\title{
METAL ION SEQUESTRATION: AN EXCITING DIMENSION FOR MOLECULARLY IMPRINTED POLYMER TECHNOLOGY
}

\author{
DMS Mosha and LL Mkayula \\ Chemistry Department, University of Dar es Salaam \\ P. O. Box 35061, Dar es Salaam Tanzania
}

\begin{abstract}
The use of a tight binding macrocyclic ligand to complex a metal ion so that this serves as receptee on the Molecularly Imprinted Polymer (MIP) receptor as described here affords a sequestration route for a targeted metal ion, with potential for environmental remediation and restoration applications. Ethylene glycol dimethacrylate (EGDMA) based co-polymer systems with either acrylamide or 4-vinyl pyridine as binding monomers are examined. These monomer choices provide strong non-covalent interactions with complementary structural elements in the MIP. The nickel(lI) ion whose chemistry is a reasonable substitute for many of the common environmentally important metal ions was examined as prototype. With ( $N, N^{\prime}, N^{\prime \prime}, N^{\prime \prime \prime}$-tetra(2-carbamoylethyl)1,4,8,11-tetraazacyclotetradecane)nickel(lI) perchlorate, A4cyclamNi(CI04h serving as imprint, the MIP's have yielded competitive rebinding affinities (17 - 95\%) excelling traditional documented levels for noncovalent interactions with magnitudes dependent on monomer identity and rising with its content. Suppressed relative rebinding magnitudes apply for non-templated 'blind' polymer controls. For the 4-vinyl pyridine case at the imprint: monomer level of 1:2 and 1:6. the contrasted MIP vs. control values are $17 \%$ vs. $14 \%$ and $95.6 \%$ vs. $35.6 \%$ respectively while for acrylamide monomer at the 1:4 and 1:12 imprint: monomer levels, the corresponding magnitudes are $18 \%$ vs. $16 \%$ and $74.4 \%$ vs. $21.2 \%$ respectively. This validates an imprinting effect, which gains prominence at higher monomer excesses. These polymer materials were shown to be robust and durable even under severe heat stress conditions. Evaluation of durability in terms of thermal stress $\left(120^{\circ} \mathrm{C}\right.$; 24h) for the 4-vinyl pyridine MIP (imprint: monomer = 1:2) yielded an affinity improvement from 17 to $26 \%$. For re-use operations, affinity increments to 19 , $23,24,27$, and $31 \%$ were recorded for five re-cycles.
\end{abstract}

\section{INTRODUCTION}

Following their discovery (Wuff and Sarhan 1972, Wuff 1995) molecularly imprinted polymers (MIP's) have seen rapid monumental expansion in terms of applications in such fields as racemate resolution, artificial antibody mimics, chemosensor technology, selective catalysis, artificial enzymes, targeted drug delivery systems, demanding synthesis or separation protocols and numerous unfolding scientific applications as testified by the many patents the field continues to generate (Arnold and Dhal 1992, Hedborg et al. 1993, Walton et al. 1997). Scientific knowledge regarding the mechanism, molecular level phenomenon and fundamentals involved in recognition and imprinting has likewise expanded. The technique involves the preparation of a polymer in the presence of a print molecule (guest) whereby specific interactions are obtained between functional monomers and the imprint. The product polymer, after template removal thus contains the specific chemical "memory" in sites that selectively and discriminately recognize the imprint and are thus capable of its reuptake. The MIP-to-imprint interaction types are covalent and or non-covalent, with the later often a preferred mode in an increasingly large number of applications (Nishide and Tsuchida 1976, Kuchen and Schram 1988, Ramstrom et al. 1993, Sellegren and Shea 1993, Chen et al. 1997, Garcia et al. 1998, Sellegren 2000) on account of the rapid 
reversible uptake it affords and the similarity to natural systems such as enzymes and antibodies. The hydrogen bonding and ligation interactions examined here for acrylamide and the weakly basic vinyl pyridine monomer respectively fall into this category. The imprint is a tightly bound metal ion complex of a tetraaazomacrocycle; $\left\{\mathrm{N}, \mathrm{N}^{\prime}, \mathrm{N}^{\prime}, \mathrm{N}^{\prime \prime}\right.$ tetra $(2-$ carbamoylethyl)-

1,4,8,11 tetraazacyclotetradecane $\}$ nickel(II) perchlorate, A4cyclam $\mathrm{Ni}(\mathrm{CI04}) 2$ (1). It s rational structural features capitalize on the inherent superior metal binding capability of tetraaazomacrocycies (Wainwright 1980, Eyring and Petrucci 1990, Busch 1993). The four pendant arms which terminate in amide functions afford multiple interactions with complementary amide functions on the polymer (from acrylamide (2) leading to special optimized four-centre $2 \mathrm{H}$-hydrogen bonds $\mathbf{3}$ expected to confer superior effectiveness beyond the traditional single two-centre H-bond version. Vinyl pyridine monomers 4 coordinate as appropriate to the metal centre via the nitrogen donor as shown in 5. Rigidity of the polymer framework calls for a high content of ethylene glycol dimethacrylate (EGDMA) cross linker 6 and use of a suitable noncompeting solvent to render such polymers macroporous for rapid access of sites during rebinding equilibria. Representations of the bound imprint interacting with acrylamide monomers and the MIP recognition site are as in $\mathbf{7}$ and $\mathbf{8}$.

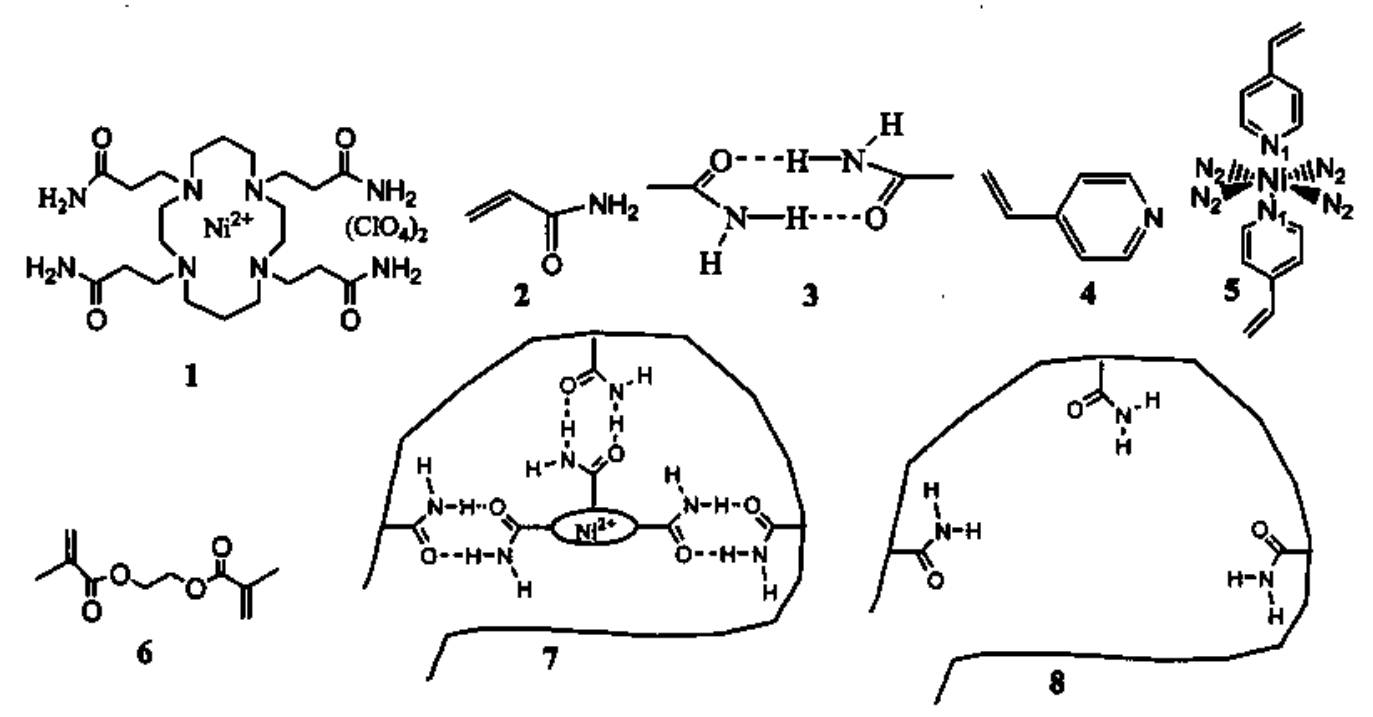

Noncovalent MIP's have traditionally yielded lower rebinding capacities, often below $20 \%$ of the theoretical sites available (Ramstrom et al. 1993, Sellegren and Shea 1993, Wuff 1995) as opposed to their covalent counterparts (values $>80 \%$ ). The rational structural elements in the MIP receptee afford multi-point (x4) H-bonding interactions in the case of acrylamide or $\mathrm{x} 2$ in the vinyl pyridine case that enhance the effective selectivity and affinity. A synergistic bimodal combination incorporating the two distinct monomers in the same MIP will be the subject of a later 
report. Additionally this 'receptor within a receptor' approach involving two recognition events; i) metal ionlligand step and ii) the resulting metal complex/polymer binding site step caters for enhanced overall effective selectivity. The target application, the sequestration of contaminant metal ion for environmental remediation and restoration, mimics nature's own mechanism and ground rules whereby certain soil bacteria secrete powerful enzymes (called siderophores) which dissolve and capture iron from chemically bound soil sources, and convey the resulting complex to the cell wall for recognition and admission (Telford and Raymond 1995). These roles are mimicked in this approach by the macro cyclic ligand and the templated polymer respectively. Two opportunities for the application of selectivity are thus; I) ligand recognizes the metal ion and 2) the resulting complex is recognized by the binding site on the MIP thus affording enhanced effectiveness and selectivity even in competitive of circumstances. Nickel(II) has been chosen as a prototypic contaminant metal ion for these investigative protocols due to the simplicity and certainty with which its complex chemistry can be treated, the kinetic inertness of the square planar complexes it forms with tetraazamacrocycles plus the reasonable reproducibility it affords for many environmentally important metal ion contaminants. The noncovalent imprinting approach holds promise in environmental contaminant metal ion remediation and restoration applications where a prototypic soil poultice would entail adding the MIP to the irrigated test soil in the presence of the ligand which would capture the targeted metal ion for transfer to the MIP receptor sites. Recycle operations would then entail extraction of the loaded MIP to obtain a concentrated waste form for disposal.

\section{EXPERIMENTAL}

All starting materials supplied from commercial sources were used without further purification. Azo(bis )isobutyronitrile was supplied by A TOFINA Chemicals Inc.,

USA, methyl cyanide was supplied by $\mathrm{M} \mathrm{S}$ D S, methyl alcohol, acrylamide, $\mathrm{N}, \mathrm{N}^{\prime}, \mathrm{N}^{\prime}, \mathrm{N}$ "'-tetra(2-carbamoylethyl) 1 ,4,8,11-tetraazacyclotetradecane

(A4cyc1am), nickel(II) perchlorate hexahydrate, diethyl ether were supplied by BDH Laboratory Supplies, Poole, BHI51.TD England, Nitric acid 99.999\% was supplied by ex Aldrich, USA.

\section{MIP preparations}

Polymerizations were carried out in sealed tubes at the $1: 4: 20$ imprint : monomer: crosslinker level at $50^{\circ} \mathrm{C}(24 \mathrm{~h})$ after degassing with argon both before and after addition of the initiator azo(bis)isobutyronitrile (AIBN). A I: I $\mathrm{MeCN} / \mathrm{MeOH}$ solvent mixture to the extent of $\mathrm{I} \mathrm{ml}$ per $\mathrm{g}$ of solid was added as porogen. At completion, tubes were crushed and product dried at R.T. under vacuum (12 h) to remove porogen then ground and sieved to a $75-125 \mu \mathrm{m}$ diameter working particle size. After a $24 \mathrm{~h}$ soxhlet extraction $(\mathrm{MeCN})$ and vacuum drying (R.T.-12 h) the material is ready for rebinding equilibria. The control polymer preparation is exactly similar to its imprinted counterpart but omitting imprint.

\section{Synthes is $\quad$ of $\quad$ th e tetraazomacrocyclic ligand}

N,N',N",N"'-tetra(2-carbamoylethyl)-

1,4,8,11-tetraazacyclotetradecane (A4cyclam) was synthesized from cyclam and acrylamide, according to literature methods (Freeman et al. 1984). Acrylamide (12.63 g, $177 \mathrm{mmol}$ ) was dissolved in 150 $\mathrm{ml}$ of methanol and filtered. Solid cyclam 
(5.08 $\mathrm{g}, 25.3 \mathrm{mmol})$ was added to the filtrate. The reaction mixture was refluxed for $48 \mathrm{~h}$ and then cooled to room temperature. The white precipitate that formed was collected by filtration, washed with ethanol and dried in vacuo. Further recrystallization from ethanol/water (5/1) afforded an analytically pure product. Yield: $6.2 \mathrm{~g}(50 \%)$. Purity was ascertained by elemental and spectral (NMR) analysis.

A4cyciam Ni(CI0 $)_{2}$ (1)

A variant of the literature methods (Wainwright 1980, Wainwright 1983) was adapted for the purpose of preparing compound (1). CAUTION! Perchlorates present a potential explosion hazard and should be handled with care. A4cyclam (1.62 g. $3.34 \mathrm{mmol}$ ) was dissolved in 50 $\mathrm{ml}$ of water and heated to reflux. Nickel(II) perchlorate hexahydrate $(1.23$ g. 3.36 $\mathrm{mmol}$ ) in $5 \mathrm{ml}$ water was added dropwise with stirring and solution allowed to reflux for two minutes. The reaction mixture was rapidly cooled to room temperature in an ice bath and evaporated to dryness under a stream of air. The resulting oily blue residue was redissolved in $25 \mathrm{ml}$ of acetonitrile and filtered. Anhydrous diethyl ether was added to the filtrate with vigorous stirring until blue green tacky oil separated. After the supernatant was decanted off, the tacky oil was treated with diethyl ether while stirring to yield a blue precipitate, which was collected by filtration, washed with diethyl ether and dried under vacuum (RT). Yield: $1.69 \mathrm{~g}(68 \%)$. The product was characterized by using MS and elemental analysis.

\section{Evaluation of rebinding affinity}

A weight of polymer (26 mg) was equilibrated with a $0.2 \mathrm{M} \mathrm{MeCN}$ solution of the imprint ( $24 \mathrm{~h})$ to effect rebinding. The loaded polymer was filtered and washed with chilled $\mathrm{MeCN}$ (dry ice) and vacuumed
(RT) to expel solvent. A weighed amount was then extracted (12 h) with $99.999 \%$ pure grade nitric acid, and extract assayed for $\mathrm{Ni}(\mathrm{II})$ by Inductively Coupled Plasma Optical Emission Spectroscopy (ICP-OES). Similar extraction of the MIP for which no rebinding has occurred furnishes an imprint residual content designating that in inaccessible polymer sites.

\section{Evaluation of polymer durability}

To assess thermal stress durability, a weight of unloaded MIP (26 mg) was heated at $120^{\circ} \mathrm{C}(48 \mathrm{~h})$ and subsequently equilibratedas appropriate to rebind imprint for affinity evaluation.

To evaluate affinities during re-cycles, the following procedure was adopted:

A weight of unloaded MIP (26 mg) was equilibrated with the $\mathrm{MeCN}$ solution of the imprint as per affinity evaluation protocols above and the extracted material retained on a sinter filtration medium. After several $\mathrm{MeOH}$ rinses and drying under vacuum (RT- $4 \mathrm{~h}$ ), the MIP was subjected to the rebinding process to determine the new affinity. The extracted material was reretained as described above for subsequent repeats as necessary. A slight yellow shade was seen to develop with progress of recycle.

\section{RESULTS AND DISCUSSION}

Increasing rebinding monomer content in MIP's has a striking effect on the rebinding affinity determined as expressed above. For the case of acrylamide monomer, the starting value of $18 \%$ at the no excess molecularity (imprint : monomer $=1: 4$ ) represents an encouraging performance for this class, excelling traditional noncovalent imprinting affinities (Sellegren, et at. 1988, Sellegren and Shea 1993, Wuff 1995). The no excess molecularity being the stoichiometric ratio of the imprint to the 
monomer as alluded to above. The affinity rises by about a fourfold to $78 \%$ at the threefold (imprint: monomer $=1: 12$ ) excess, Figure 1. Care is necessary with higher monomer excesses. The lower crosslinking values they entail may compromise the rigid macroporous polymer framework for which the literature guidelines for MIP's generally indicate $a \geq$
$80 \%$ crosslinking value for effective retention of recognition. All polymerization runs conform to this guideline. For the case of 4-vinyl pyridine, as levels rise from 1:2 (a no excess molecularity) to a generous $1: 6$, the rebinding capacity rises from barely detectable to $95 \%$. (Fig. 2).

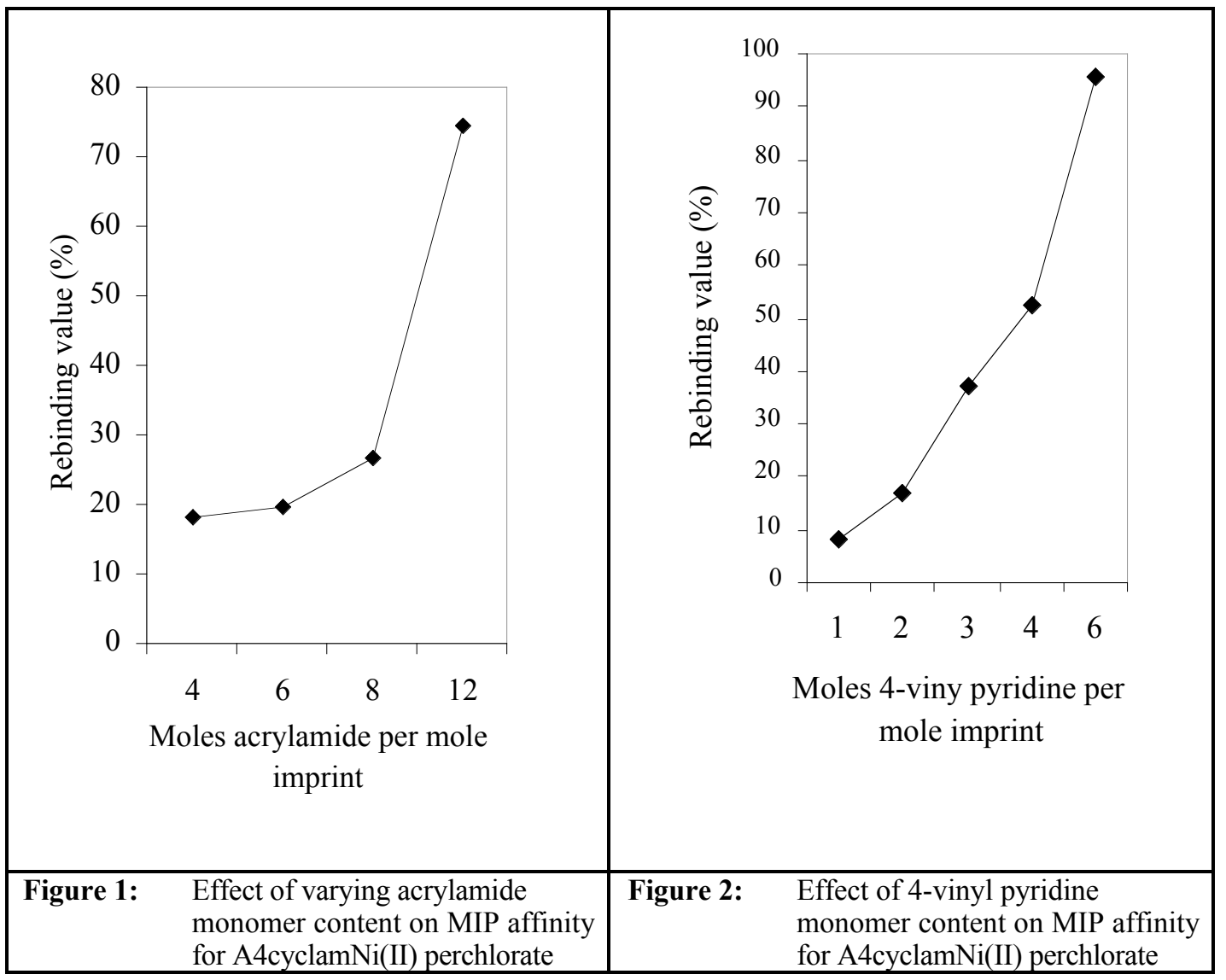

The most dramatic affinity surges occur with excess binding sites for both monomers. The later rebinding affinities are some of the highest on record for this class of materials and are supportive of binding site excess as a viable MIP optimization strategy capable of record affinities. An imprinting effect is valid for all test MIP's as validated by affinity data for imprinted polymers always excelling the blank controls - see table. The work of Wuff and others (O'Shannessy, et al. 1989, Sellegren and Shea 1993, Wuff 1995) has underpinned the importance of generous monomer excesses for enhanced imprinting performance. Optimization, stabilization 
Mosha \& Mkayula - Metal ion sequestration ...

and homogenization of pre-polymerization binding site assemblies and molecularities are favoured by, among others, generous binding monomer excess, low polymerization temperature and strong interacting monomer choices (O'Shannessy et al. 1989, Sellegren and Shea 1993, Wuff 1995).

Table 1: Affinities of imprinted MIP's versus their controls to probe imprinting at varying acrylamide and 4-vinyl pyridine monomer levels.

\begin{tabular}{cccccc}
\hline $\begin{array}{c}\text { Binding } \\
\text { monomer }\end{array}$ & $\begin{array}{c}\text { Imprint to } \\
\text { monomer } \\
\text { ratio }\end{array}$ & $\begin{array}{c}\text { Polymer } \\
\text { (label; type) }\end{array}$ & $\begin{array}{c}\text { Cross- } \\
\text { linking }\end{array}$ & $\begin{array}{c}\text { Capacity } \\
(\boldsymbol{\%})\end{array}$ & $\begin{array}{c}* \text { Affinity } \\
\text { ratio } \\
\text { (a)/(b) }\end{array}$ \\
\hline \multirow{3}{*}{ 4-vinyl } & $1: 6$ & $\mathrm{P} 34(\mathrm{a})$ & 85.6 & 95.6 & \\
pyridine & & $\mathrm{P} 34(0)(\mathrm{b})$ & 85.6 & 35.6 & 2.7 \\
\cline { 2 - 6 } & $1: 2$ & $\mathrm{P} 31(\mathrm{a})$ & 97.3 & 17 & \\
& & $\mathrm{P} 31(0)(\mathrm{b})$ & 97.3 & 14 & 1.2 \\
\cline { 2 - 6 } Acrylamide & $\mathrm{P} 53(\mathrm{a})$ & 82.3 & 74.4 & 3.5 \\
\cline { 2 - 6 } & $1: 12$ & $\mathrm{P} 53(0)(\mathrm{b})$ & 82.3 & 21.2 & 1.1 \\
\hline
\end{tabular}

* (a) = Imprinted polymer (b) = Control

Comparing data on the affinity of selected MIP's against the appropriate blanks (controls) furnishes useful indications as to the selectivity. Use of such data for exploring this variable (Table 1) indicates a favourable imprinting environment with higher monomer excess ranges. Scrutinizing Table 1 verifies that improvements of magnitudes from 1.2 and 1.1 (MIP's versus blanks) are obtained in low ranges to 2.7 and 3.5 for the respective cases of 4-vinyl pyridine and acrylamide at high monomer excess. This is an added testimony of the essence and rationale for inclusion of adequate excess for which Sellegren and Shea (1993) and Wuff (1995) have recommended a three - to four - fold excess. The commonest binding monomers in use in imprinted polymer practice are methacrylic and acrylic acids undoubtedly on account of ready availability, cheapness and compatibility for electrostatic and Hbonding interactions with many naturally occurring substances compatible with the technology (e.g. Sellegren et al 1985, Sellegren and Shea 1993, Wuff 1995, Sellegren 2000). Reports examining the use of acrylamide in the literature are restricted and the target here is to document the imprinting capability of its unique Hbonding interaction with amide functionalities on imprints. Several reports cover the vinyl pyridines (e.g. Ramstrom et al 1993, Sellegren 2000) registering equal imprinting effectiveness for the 2- or 4 isomer. Kuchen and Schram (1988) unequivocally specify the 4-isomer for examining cross-linking polymerization of monomeric or oligomeric complex forming compounds in the presence of metal ions. Thus identification of optimal interacting monomers is critical and is dictated by the system. The 2- and 3-vinyl pyridine isomers, to be examined in a later report, will examine informative contrast for monomer structural requirements for this system.

The polymers have proven to be very durable in more aggressive conditions than are likely to be found in application environments. Severe thermal stress $\left(120^{\circ} \mathrm{C} ; 24 \mathrm{~h}\right)$ for the 4-vinyl pyridine MIP (imprint: monomer $=1$ :2) surprisingly 
yields affinity improvement from 17 to $26 \%$. Recycle operations (inclusive of repeated imprint stripping requiring $\mathrm{HNO}_{3}$ ) has not diminished performance but elicited enhancements to $19 ; 23 ; 24 ; 27 ; 31 \%$ in five re-cycles. While the exact molecular level origin of these interesting and useful phenomena remains pending, the outcome certainly confers a desirable robustness and durability, on the test MIP's.

\section{CONCLUSION}

Optimized non-covalent imprinting involving (i) generous monomer excess (ii) multi-point binding strategies and (iii) careful choices of monomers can produce MIP's possessing phenomenal molecular recognition potential and record affinities uncommon with this category. Since the imprint is the complex of a metal ion with a tight binding macrocyclic ligand possessing rational structures for strong interaction with the MIP types investigated, the approach affords a novel sequestration strategy for environmentally important contaminant metal ion analogues. The two stage recognition phenomenon is well suited for contaminant metal ion remediation and restoration applications, as it is compatible with the aquatic environments encountered in common pollution scenarios. Additionally, the test ligand and metal complex are water soluble, a prerequisite for viability of the approach. The observed durability of the MIP materials as regards thermal stress and recycling is an added asset given the demanding environments targeted for their application

\section{REFERENCES}

Alunni S, Laureti V, Ottavi Land Ruzziconi R 2003 Catalysis of the (3-elimination of HF from isomeric 2fluoroethylpyridines and I-methl2 fluoroethylpyridinium salts.
Protonactivating factors and methylactivating factors as a mechanistic test to distinguish between concerted E2

and E1cb irreversible mechanisms. J. Org. Chem. 68: 718725.

Arnold FH and Dhal PK 1992 Metal coordination interactions in thetemplate-mediated synthesis of substrate-selective polymers: Recognition of bis(imidazole) substrates by copper(II) iminodiacetate containing polymers. Macromolecules 25: 7051-7059.

Busch DH, Collinson SR and Hubin TJ 1998 International Patent Publication under PCT, WO 98/39098, Sept. 11.

Chen H, Olmstead MM, Albright RL, Devenyi J. and Fish RH 1997 Metalion-templated polymers: Synthesis and structure of N-(4vi n y 1 b e n z y 1) 1, 4, 7 triazacylononanezinc(II)

complexes, their copolymerization with divinylbenzene, and metal-ion selectivity studies of the demetalated resins-Evidence for sandwich complex in the polymer matrix. Angew. Chem. Int. Ed. Engl. 36: 642645.

Eyring, EM and Petrucci S 1990 In: Inoue $\mathrm{Y}$ and Goekel GW (eds) Cation Binding by macrocycles, Marcel Dekker, Inc., New York.

Freeman MG, Barefield K and Van Derveer DG 1984 Studies on Nickel(II) complexes of cyclan ligands containing functionalized nitrogen substituents: Synthesis, isomerization, and N-dealkylation. Inorg Chem. 23: 3092-3103.

Garcia R, Pinel C, Madic C, and Lemaire M 1998 Ionic imprinting effect in gadolinium/lanthanum separation. 
Tetrahedron Lett. 39: 8651-8654.

Hedborg E, Winquist F, Lundstrom I, Andersson LI and Mosbach K 1993 Some studies of molecularly imprinted polymer membranes in combination with field-effect devices. Sensor. Actuator. A-Phy. 36-38: 796-799.

Kuchen W and Schram J 1988 Metalionselective exchange resins for matrix imprint with methacrylates. Angew. Chem. Int. Ed. Engl. 27: 1695-1697.

Nishide N and Tsuchida E 1976 Selective adsorption of metal ions on poly( 4vinlypyridine) resins Makromol. Chem. 177: 2295-2310.

O'Shannessy DJ, Ekberg, B. and Mosbach, K. 1989 Molecular imprinting of amino acid derivatives at low temperature $\left(\mathrm{O}^{\circ} \mathrm{C}\right)$ using photolytic homolysis of azobisnitriles. Analytical Boichemistry 177: 144149.

Ramstrom O, Anderson Land Mosbach K 1993 Recognition sites incorporating both pyridinal and carboxy functionalities prepared by molecular imprinting. J. Org. Chem. 58: 76627664.

Sellergren B and Shea K 1993 Influence of polymer morphology on the ability of imprinted network polymers to resolve enantiomers. J. Chromatogr. 635: 31-49.

Sellergren B 2000 Imprinted polymers with memory for small molecules, proteins, or crystals. Angew. Chem. Int. Ed. Engl. 39: 1031-1037.

Sellergren B, Lepisto M and Mosbach K
1988 Highly enantioselective and substrate-selective polymers obtained by molecular imprinting utilizing noncovalent interactios, NMR and chromatographic studies on the nature of recognition. J. Am.. Chem.Soc. 110: 5853-5860.

Telford JR, Raymond KN 1996 In: Gokel, G (ed) Supramolecular Chemistry, Pergamon, Oxford.

Wainwright KP 1980 Chemistry of structurally developed macrocycles. Part 1. Complexation properties of $\mathrm{N}, \mathrm{N}^{\prime}, \mathrm{N} ", \mathrm{~N}$ "'-tetra(2-cyanoethyl) 1 ,4,8,11-tetra-aza-cyclotetradine with nickel(II). J. Chem. Soc. Dalton Trans. 2127-2120.

Wainwright KP 1983 Chemistry of structurally developed macrocycles. Part 2. Synthesis and complexing properties of N,N',N",N"'tetra(3aminopropyl)-1,4,8,11-

tetraazacyclotetradecane with nicke(II). J. Chem. Soc. Dalton Trans. 1149-1152.

Walton PH, Saunders GD, Joyce M. and Port SN 1997 UK Patent Application no. 979946.7.7.

Wuff G and Sarhan A 1972 The use of polymers with enzyme-analogue structures for resolution of racemates. Angew. Chem. Int. Ed. Engl. 11: 341.

Wuff G 1995 Molecular Imprinting in crosslinked materials with the aid of molecular templates-A way towards artificial antibodies. Angew. Chem. Int. Ed. Engl 34: 1812-1832. 


\section{INSTRUCTIONS TO AUTHORS}

The Tanzania Journal of Science is published by the Faculty of Science, University of Dar es Salaam. Contributions on original work are accepted in the fields of pure and applied science as full research papers or notes (short communications). Comprehensive reviews and book reviews will be accepted at the discretion of the editor. Authors are requested to strictly follow the instructions below.

\section{Submission}

1.1 All papers should be submitted in triplicate hard copies and an electronic version must be included.

1.2 Submission of a paper implies that it has not been published previously, and is not simultaneously under consideration for publication elsewhere.

1.3 All editorial correspondence should be addressed to:

The Chief Editor, Tanzania Journal of Science, University of Dar es Salaam, P.O. Box 35065, Dar es Salaam, TANZANIA.

E-mail: TJS@Science.udsm.ac.tz

\section{Manuscript preparation}

2.1 Manuscripts must be typewritten, double spaced and with margins of at least $2.5 \mathrm{~cm}$ on the top and left side of the papers.

2.2 Use font Times New Roman 12

2.3 Limit your manuscripts to a maximum of 15 typed pages of A4 size including illustrations (e.g. photographs, diagrams, tables). The maximum length for short communications will be 6 typed pages

\section{Figures and tables}

3.1 Figures and tables should appear in numerical order and be described in the body of the text.

3.2 Originals of all figures should be in black ink and all lettering should be bold, readable, and big enough to allow reduction for final layout. Use $11 / 2 \mathrm{pt}$ line thickness for line drawings.

3.3 Figures, tables and other illustrations must be placed separately at the end of the paper. Indicate in pencil in the margins, recommended positions of figures, chemical schemes and tables in the text. An example of table layout is shown in Table 1.

3.4 Figure captions should be written on a separate sheet, but table titles should be typed on the same page as the table.

Table 1: Dimensions of text area to be used for Tanzania Journal of Science articles and of international and US paper sizes

\begin{tabular}{cccccc}
\hline & Text area & \multicolumn{2}{c}{ A4 paper } & \multicolumn{2}{c}{ US (Imperial) paper } \\
\cline { 3 - 6 } & $(\mathrm{mm})$ & $(\mathrm{mm})$ & (in) & $(\mathrm{mm})$ & (in) \\
\hline Depth & 270 & 297 & 11.69 & 279.32 & 11.0 \\
Width & 185 & 210 & 8.27 & 215.84 & 8.5 \\
\hline
\end{tabular}

\section{Units}

4.1 The International System of Units (SI units) must be used throughout. Equivalent units e.g. pounds, miles, may be given in parentheses after the appropriate SI unit. 


\section{Manuscript content}

Manuscripts should contain the following parts:

\subsection{Title}

This must be short and precise. Abbreviation are not allowed. The title will be followed by the author(s) name(s), postal and E-mail addresses.

\subsection{Abstract}

This should be a brief summary of what was done, the main findings and the conclusions drawn. The abstract should not exceed 200 words. All papers, including short communications must have abstracts.

\subsection{Introduction}

This should contain a brief survey of the relevant literature and the objectives of the work.

\subsection{Materials and Methods}

Sufficient information should be given here, including relevant references, so that the study can be repeated by someone else.

\subsection{Results}

All illustrations must bear full captions, and must be specifically mentioned in the text, e.g. potassium ions enhanced stomata opening (Fig.5). The same data should not be presented both in the form of a graph and a table. Shorter tables and graphs should be combined to save space.

\subsection{Discussion}

This should emphasize the significance of the results and their relationship with other published work, and with the original objectives of the investigation. A brief concluding statement and recommendations for further work are normally given in the final paragraph.
The section on results and that on discussion may be combined to give one section on "results and discussion."

\subsection{Acknowledgements}

This should be very brief, about one to two sentences.

\subsection{References}

5.8.1 Citation in text

5.8.1.1 Use surname of author and year of publication: John (2000) or (John 2000).

5.8.1.2 Different references cited together should be in date order, for example: Mark 1970, Peter and Suleiman 1998, Green 2000).

5.8.1.3 If a paper has been accepted for publication but has not been published the term "(in press)" should be used instead of a date.

5.8.1.4 The abbreviation "et al." should be used in the text when there are more than two co-authors of a cited paper, for example: Maharage et al. (2001) or (Maharage et al. 2001).

5.8.2 List of references

5.8.2.1 References should be listed alphabetically at the end of the paper. Only the literature actually cited in the text should be listed here.

5.8.2.2 In the case of journals, authors' names followed by initials, the year of publication the title of the article, the name of the journal, the volume and the first and last pages of the article should be given in that order, for example:

a ) Playne MJ 1984 Increased digestibility of bagasse by pretreatment with alkali and steam explosion. Biotechnology Bioeng. 26:426-433.

b) Hecky RE, Mugidde R, Twongo T, Balirwa J and Mavuti K 2000 Ecosystem change in Lake Victoria. Tanzania J. Science 28: 50-61.

5.8.2.3 Book reference styles: a) standard, b) article in a book, c)thesis

a ) Schlegel HG 1988 General Microbiology. $6^{\text {th }}$ edn, Cambridge University Press, Cambridge.

b ) B oyton W 1984 Cosmochemistry of the rare earth 
elements: Meteorite studies. In: Henderson P (ed) Rare earth element geochemistry Elsevier, Amsterdam.

c) Tamminen T 1990 Eutrophication and the Baltic sea: Studies on phytoplankton, bacterioplankton and pelagic nutrient cycles. $\mathrm{PhD}$ thesis, Department of Environmental Conservation, University of Helsinki. 